\title{
Saudi neurosurgery residency and COVID-19: How are we coping?
}

Razan M. Almufarriji, MD, Abdullab O. Alobaid, MD, FRCSC, Fabd A. Alsubaie, MD.

The first case of Coronavirus Disease-19 (COVID-19) in Saudi Arabia was diagnosed in the eastern province on the 2 nd of March $2020 .{ }^{1}$ A state of global pandemic was declared on the 11 th of March. ${ }^{2}$ Since then, the pandemic has had an extensive impact on all aspects of society, including postgraduate medical education and training.

The workflows at the Department of Neurosurgery have been adjusted based on the recommendations of the Ministry of Health and of the Saudi Association of Neurological Surgery. ${ }^{3}$ The modifications include the following: 1) the cancelation of all elective surgeries except preplanned neuro-oncological procedures. There were decreases of approximately $20 \%, 50 \%$ and $90 \%$ in surgical cases during the months of March, April and May, respectively (Hospital registry). 2) The regularly offered neurosurgical services were replaced by an on-call team consisting of a junior resident, a senior resident and an attending consultant. There are 2 of these teams, and they alternate every 2 weeks to maintain business continuity in case of unprotected exposure, isolation or an infection among the staff. 3) All clinical and academic meetings were suspended. 4) A designated COVID-19 team responsible for managing suspected or confirmed COVID-19 cases was established. This team consists of board-certified physicians only. 5) Outpatient clinics continued to operate through virtual methods on a regular schedule, and patients were contacted by phone and managed accordingly. Patients who needed further in-hospital assessments or emergency visits were managed through patient relation (PR) personnel who instructed, arranged and escorted them to a clinic. Medications were prescribed and mailed to patients if needed. The
Johns Hopkins neurosurgical department reported the use of video clinic visits during the pandemic, which greatly facilitated the process of establishing patients' medical histories and conducting examinations. ${ }^{4}$ 6) Hospital staffs including residents were offered on-campus accommodations as optional alternatives for their own residence. 7) Due to uncertainty of the situation in some regional hospitals, many residents' rotations were declined. Consequently, many residents had their training plans abruptly modified.

Unfortunately, this severe shortage in residents' clinical and educational activities was associated with limited alternatives. Residents continued some of their theoretical learning objectives in online-based webinars hosted by local and international neurosurgical societies. A survey was distributed to 115 neurosurgical residents at different levels to assess the impact of COVID-19 on their training and wellbeing. There was 57\% dissatisfaction towards teaching status, mainly due to the scarcity of hands-on practice, randomly organized webinars and lack of infrastructures for optimal on-line teaching for most residents. The same survey showed a decrease in surgical exposure of more than $64 \%$ of participants, from 3-4 cases per week to $0-1$ cases per week.

These unprecedented changes and the uncertainty of the situation have negatively impacted residents' wellbeing. The majority of the surveyed residents $(66 \%)$ indicated that their fear of spreading the virus to their families was the factor that contributed the most to their anxiety. This was followed by an ambiguity of promotion and evaluation measures, a lack of proper education, uncertainty regarding the time frame of the pandemic and, finally, an expectation of being called to cover intensive care units or quarantine areas.

From the Department Neurosurgery, King Fahad Medical City, Riyadh, Kingdom of Saudi Arabia. Address correspondence and reprint request to: Dr. Razan M. Almufarriji, Department of Neurosurgery, King Fahad Medical City, Riyadh, Kingdom of Saudi Arabia. E-mail: Razanmoh93@gmail.com ORCID ID: https://orcid.org/0000-0002-1133-7982 
During the unfolding of the pandemic, postgraduate training in neurosurgery and residents' wellbeing seem to be considerably compromised. Although there have been good intentions in terms of support from academic and governmental institutions, little has been done to remedy this situation. A comprehensive study to assess the impact of COVID-19 on training is essential. This study should be performed through 360-degree evaluations of trainees, trainers' perspectives and coping measures over the short- and long-term.

Acknowledgement. We would like to thank American Journal Experts (https://www.aje.com/) for their English editing services.

\section{References}

1. Yezli S, Khan A. COVID-19 social distancing in the Kingdom of Saudi Arabia: Bold measures in the face of political, economic, social and religious challenges. Travel Med Infect Dis 2020; 101692.

2. Cucinotta D, Vanelli M. WHO declares COVID-19 a pandemic. Acta Biomed 2020; 91: 157-160.

3. Bajunaid K, Sabbagh A, Ajlan A, Al-Jehani H, Alnaami I, Khormi Y, et al. Consensus Statement of the Saudi Association of Neurological Surgery (SANS) on Triage of Neurosurgery Patients During COVID-19 Pandemic in Saudi Arabia. Neurosciences (Riyadb) 2020; 25: 148-151.

4. Khalafallah AM, Jimenez AE, Lee RP, Weingart JD, Theodore $\mathrm{N}$, Cohen AR, et al. Impact of COVID-19 on an Academic Neurosurgery Department: The Johns Hopkins Experience. World Neurosurg 2020; 139: e877-e884.

\section{Clinical Practice Guidelines}

Clinical Practice Guidelines must include a short abstract. There should be an Introduction section addressing the objective in producing the guideline, what the guideline is about and who will benefit from the guideline. It should describe the population, conditions, health care setting and clinical management/diagnostic test. Authors should adequately describe the methods used to collect and analyze evidence, recommendations and validation. If it is adapted, authors should include the source, how, and why it is adapted? The guidelines should include not more than 50 references, 2-4 illustrations/tables, and an algorithm. 